\title{
Isotopically enriched nanoparticles in combination with mass spectrometry for the assessment of nanoparticle-biomolecule stoichiometries in engineered nanoassemblies
}

Mario Menendez-Miranda ${ }^{a}$, David Presa-Soto ${ }^{b}$, Alejandro Presa-Soto ${ }^{b}, J_{\text {Iosé M. Costa-Fernandez }}^{a *}$ and Jorge Ruiz Encinar ${ }^{a}$.

aDepartment of Physical and Analytical Chemistry, University of Oviedo, Julián Clavería 8, 33006, Oviedo, Spain.

bepartment of Organic and Inorganic Chemistry, University of Oviedo, Julián Clavería 8, 33006, Oviedo, Spain.

* corresponding authors: email: jcostafe@uniovi.es ; email: ruizjorge@uniovi.es 


\begin{abstract}
Determination of stoichiometries between engineered nanoparticles and species attached to their surface after chemical functionalization or interaction with biological media has been a hotspot during the last years, still unsatisfactory solved. Herein we propose a new approach only requiring for a simple mass spectrometric sulfur isotope ratio measurement in the final solution. Of course, enriched ${ }^{34} \mathrm{~S}$ isotope has to be previously introduced in the engineered nanoparticle structure. Conversely to existing methodologies, mostly based on relative analyte measurements, proposed method does not require any previous knowledge of the molecule concentration in the starting solution. Such rather generic approach can be applied to study physical and/or chemical interactions between any sulfur-containing molecule and a wide variety of engineered nanoparticles containing sulfur as well, either in their own core-shell structure or in the ligand used for their surface functionalization (e.g. any thiol-derivative). In this paper, an inexpensive standard synthetic procedure was carried out to incorporate the required stable isotopic ${ }^{34} \mathrm{~S}$ into the $\mathrm{ZnS}$ shell of CdSe quantum dots (QDs). Such isotopically enriched nanoparticles were then successfully applied to the quantitative assessment of stoichiometries between QDs and different target biomolecules, such as surface-functionalizing molecules (e.g. biotin) and proteins (e.g. bovine serum albumin). Of course, any biological conclusion and/or optimized bioconjugation conditions obtained using such isotopically enriched QDs analogues could be easily translated to nanoassemblies obtained using regular non-enriched QDs since they are chemically and physically identical.
\end{abstract}

Keywords: Mass spectrometry. Nanomaterials. Isotope ratios. Nanoparticle-biomolecule stoichiometry. 


\section{Introduction}

Quantum dots (QDs) are semiconductor nanoparticles (NPs) that have been extensively applied as targeted luminescent probes for in vivo and in vitro bioanalytical and bioimaging studies [1]. They are also currently used in more dynamic and sophisticated applications such as scaffolds in nanosensors and drug delivery or theranostics assemblies [2-4]. In such bioanalytical applications, QDs need to be previously functionalized with appropriate biomolecules (e.g. ligands, proteins) to guarantee their specific interaction with target analytes. As a matter of fact, such controlled functionalization of the QDs surface with appropriate functional ligands or proteins is a key issue that is unfortunately still far from being routinely achieved. In fact, it is widely recognized that new and reliable analytical methods are urgently demanded for this purpose [5].

Additionally, application of QDs in biological media is strongly affected by their interaction with proteins from the medium, bringing about the so called 'protein corona' associations. Once the NPs enter in the biological medium, protein adsorption onto NPs surface occurs [6]. This naturally formed biological layer plays a crucial role in the NPs physicochemical properties. The study of this 'protein corona' formation and its characterization has been a hotspot in bionanoresearch during the last years, and a lot of work has been devoted to characterize, quantify and understand this process [7-9]. In this context, Bovine Serum Albumin (BSA) has been typically selected as model protein to study the protein corona formation [10,11], and strong evidences of its importance to protect and make NPs more stable in biological media have been reported [12]. A critical parameter that characterizes such protein corona is the average number of proteins that are linked to the surface of the NP under given experimental conditions. However, adequate analytical approaches to carry out such protein:NP ratio determinations are still under demand [5]. Among the different strategies already described in the literature to estimate biomolecule:NP ratios, sulfur determination appears to be a promising choice as it is present in most proteins [9]. However, very often, $\mathrm{S}$ is also present in either the core-shell NPs ( $\mathrm{ZnS}, \mathrm{PbS}, \mathrm{CdSe} / \mathrm{ZnS})$ and/or in the functional ligands attached to the surface in order to render them water stable (e.g. dihydrolipoic acid or cysteine). This makes hard to distinguish the $\mathrm{S}$ originally present in the engineered 
$\mathrm{NP}$ from the sulfur later incorporated as functionalizing agents (i.e. biotin) or as present in the proteins constituting the 'protein corona' in biological media (i.e. BSA).

Herein we have synthesized conventional fluorescent CdSe QDs with a shell consisting of Cd/Zn sulfide isotopically enriched in ${ }^{34} \mathrm{~S}(\sim 95 \%)$, clearly different from natural sulfur where ${ }^{32} \mathrm{~S}$ accounts for almost $95 \%$ abundance. In that way, the incorporation of S-containing biomolecules to the QD environment can be mathematically computed from the S isotope ratios: ${ }^{34} \mathrm{~S}$ (mostly coming from QDs) to ${ }^{32} \mathrm{~S}$ (mostly coming from the biomolecule). Such sulfur isotope ratios can be accurately and precisely measured by inductively coupled plasma tandem mass spectrometry (ICP-MS/MS). The synthesis strategy of ${ }^{34} \mathrm{~S}$-enriched QDs $\left(\mathrm{QD}_{\mathrm{enr}}\right)$ together with the new analytical methodology proposed is illustrated in Figure 1. To produce such isotope-enriched nanotool, the corresponding S-containing reagent, used as precursor during the NP shell formation, had to be first synthesized using isotopically enriched elemental ${ }^{34} \mathrm{~S}$ as starting reagent. Then, $\mathrm{QD}_{\mathrm{enr}}$ could be synthesized and thoroughly characterized. Finally, the great potential of the $\mathrm{QD}_{\mathrm{enr}}$ has been evaluated by tackling two of the most challenging tasks nowadays in bionanotechnology. First, the quantitative determination of the protein corona population in different mixtures of $\mathrm{QD}_{\mathrm{enr}}$ and $\mathrm{BSA}$, selected as a model protein. Second, the accurate determination of the number of functionalizing molecules (biotin selected as a model in this case) intentionally incorporated to the $\mathrm{QD}_{\mathrm{enr}}$ surface, via chemical bioconjugation, under different experimental conditions.

It should be noted that although isotopically enriched NPs have been scarcely synthesized so far, they have proved to show the same physico-chemical properties as their natural analogues [13-16]. In those recent works, enriched NPs have been used as isotopic tracers in standard isotope dilution studies aimed at studying NPs behavior in complex matrices (e.g. the study of fate and transformations of NPs in sediment-slurry matrices, standard estuarine sediments, biological tissues, etc.). To our knowledge this manuscript reports for the first time a simple approach based on isotopically enriched NPs $\left(\mathrm{QD}_{\text {enr }}\right)$, to quantify precisely the ratio between those NPs and chemically bound (bioconjugated) biomolecules or alternatively, biomolecules adsorbed (protein corona) to the NPs surface. 


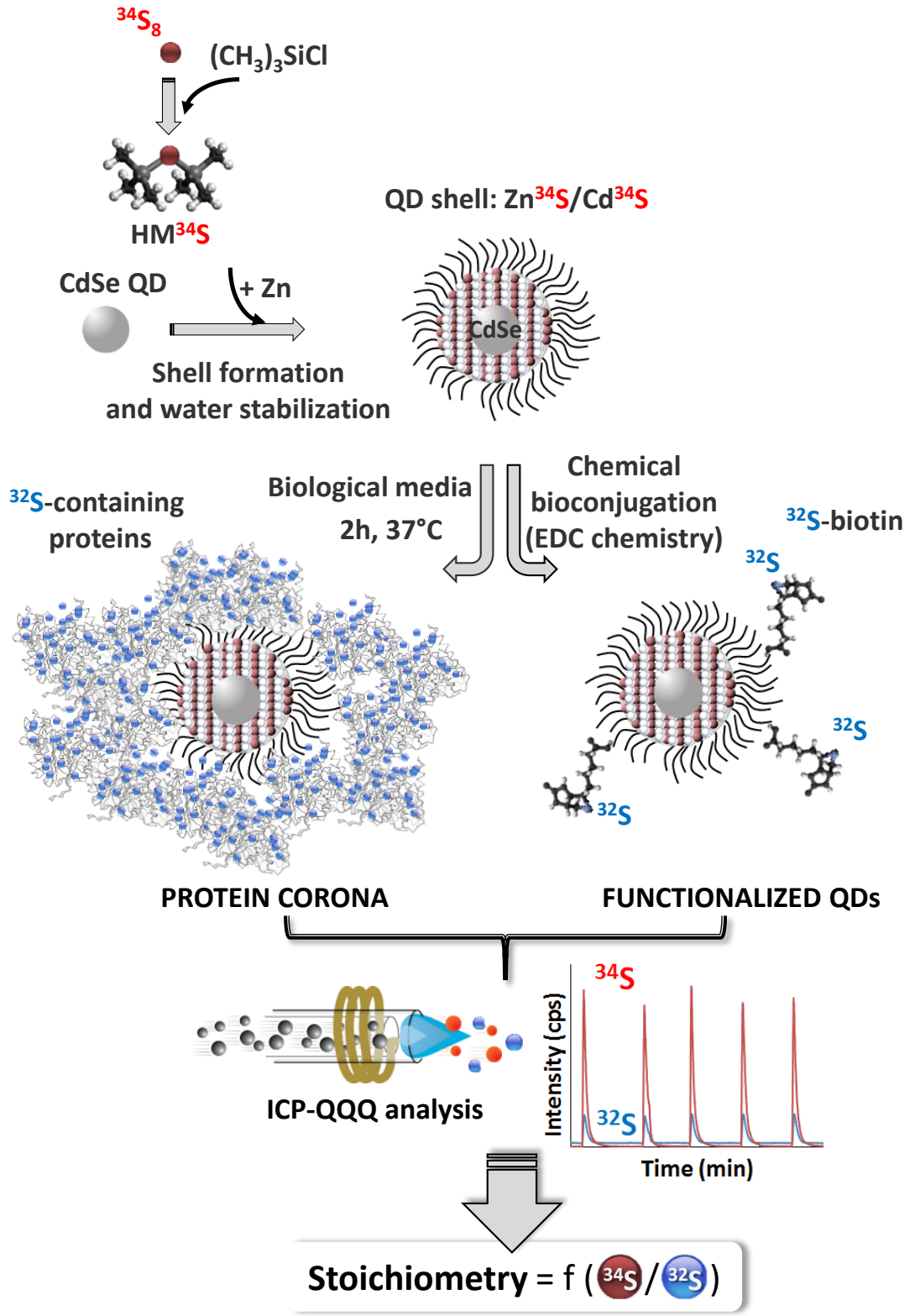

Figure 1. Schematic representation of the proposed methodology for determination of nanoparticle-biomolecule stoichiometries (molar ratios) in S-containing nanoassemblies

\section{Methods and Theory}

2.1 Synthesis and characterization of the quantum dots isotopically enriched in ${ }^{34}$ Sulfur

Standard CdSe QDs were synthesized following the organometallic route described elsewhere using $\mathrm{CdO}$ as precursor [17]. Prior to the growth of the isotopically enriched $\mathrm{Zn} / \mathrm{Cd}^{34} \mathrm{~S}$ shell over the CdSe core, 
it was necessary to synthesize and characterize the ${ }^{34} \mathrm{~S}$-enriched precursor $\left({ }^{34} \mathrm{~S}\right.$-hexamethyldisilathiane). Such compound was obtained under $\mathrm{N}_{2}$ atmosphere from the reaction of chlorotrimethylsilane with the resulting mixture of ${ }^{34} \mathrm{~S}_{8}$ with the strong reducing $\mathrm{LiHBEt}_{3}$ in organic media (see Appendix for the details).

a)

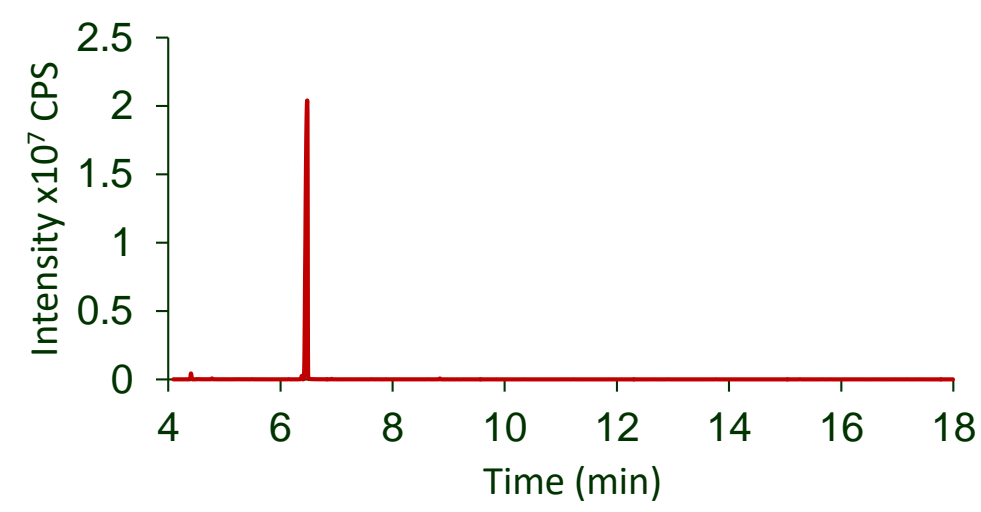

b)

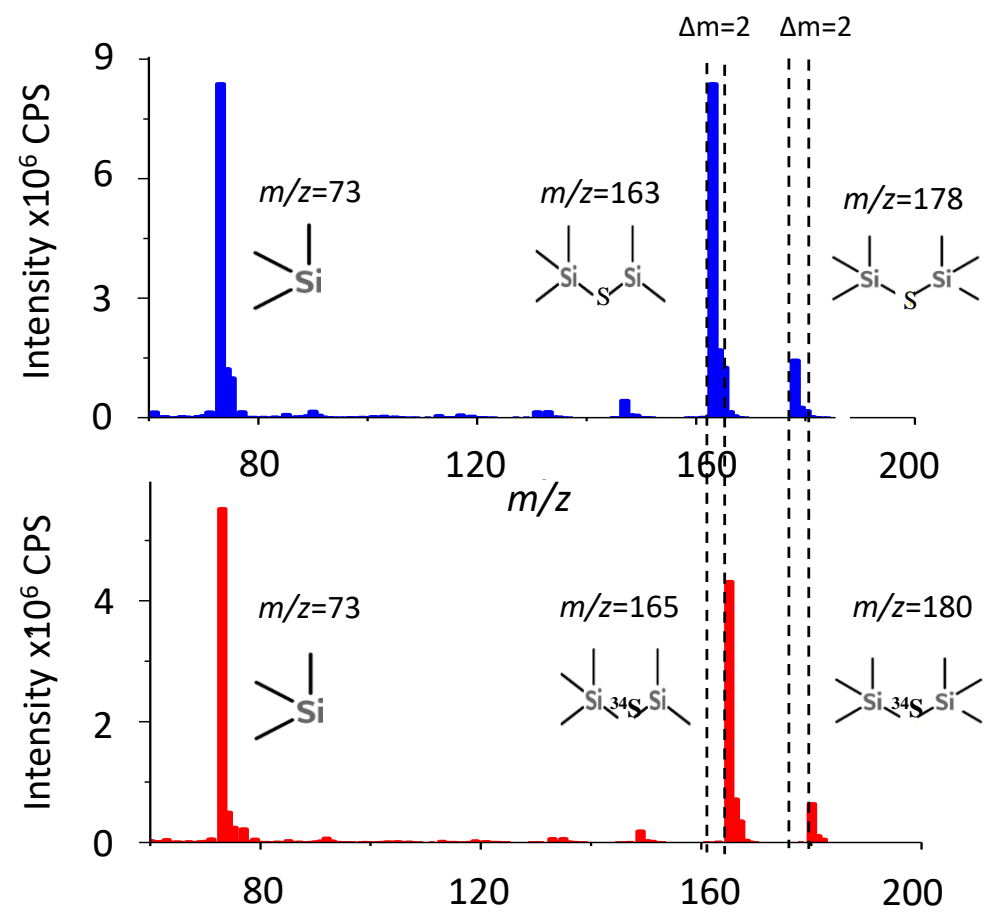

Figure 2. a) Gas chromatogram of the synthesized ${ }^{34}$ S-hexamethyldisilathiane; b) Mass spectrum of non-enriched hexamethyldisilathiane; (c) Mass spectrum (time $6.3 \mathrm{~min}$ ) of the enriched ${ }^{34}$ S-hexamethyldisilathiane. 
The product of such synthesis, was evaluated by ${ }^{1} \mathrm{H}$ and ${ }^{13} \mathrm{C}\left\{{ }^{1} \mathrm{H}\right\}$-NMR and the observed spectra are given in Fig. S1 (Appendix). Further evidences for identification and purity determination of the ${ }^{34} \mathrm{~S}-$ hexamethyldisilathiane were obtained by GC-MS. Fig. 2a shows clearly that one single peak was observed at $6.3 \mathrm{~min}$ in the GC-MS chromatogram.

Retention time matched very well with that of a commercial hexamethyldisilathiane with natural S isotopic abundance. The purity of the synthesized ${ }^{34}$ S-reagent was established to be $>99 \%$. Comparison of the Electron Ionization fragmentation pattern obtained for the ${ }^{34}$ S-enriched (Fig. 2b) and the natural abundance standard (Fig. 2c) showed the expected mass shift of 2 mass units for the molecular ion peak ( $m / z=180)$ and the sulfur containing fragment corresponding to the loss of one $-\mathrm{CH}_{3}$ group $(\mathrm{m} / z=165)$ due to the vast presence of ${ }^{34} \mathrm{~S}$ instead of ${ }^{32} \mathrm{~S}$ in the enriched and natural products, respectively. Conversely, the fragment at $m / z=73$, corresponding to $\left(\mathrm{CH}_{3}\right)_{3} \mathrm{Si}^{+}$and that does not contain sulfur, was observed in both mass spectra.

The ${ }^{34}$ S-hexamethyldisilathiane was then used for the shelling of the CdSe core previously synthetized. The so-obtained core-shell $\mathrm{QD}_{\mathrm{enr}}$ were further surface-coated with an amphiphilic polymer for aqueous stabilization (see Appendix for the details) following the procedure previously described elsewhere [18]. The size of the final $\mathrm{QD}_{\mathrm{enr}}$ was determined using transmission electron microscopy (see Fig S2, Appendix). An average $\mathrm{QD}_{\mathrm{enr}}$ diameter value of $4 \mathrm{~nm}$, with low size dispersion $( \pm 0.4 \mathrm{~nm})$ was obtained. The UV-Vis absorbance and fluorescence spectra of the synthesized $\mathrm{QD}_{\text {enr }}$ are shown in Figure S3 (Appendix). As can be seen, the use of the isotopically enriched ${ }^{34}$ S-precursor did not affect the photoluminescent properties of the resulting NPs. This is in agreement with other reports showing that isotopically enriched NPs show the same physical and chemical properties as their natural analogues $[13,16]$.

\subsection{Elemental characterization of water-soluble $Q D_{\text {enr. }}$}

After polymer coating of the $\mathrm{QD}_{\mathrm{enr}}$, several purifications were carried out. First, samples were passed through syringe filters $(0.22 \mu \mathrm{m})$ (Teknokroma, Spain) to remove eventual nanoparticle aggregates generated during the $\mathrm{QD}_{\mathrm{enr}}$ solubilization process. Second, buffer was exchanged by ultrapure water using 
two rounds of dilution and preconcentration through a centrifuge filter (molecular weight cut-off $=100$ $\mathrm{kDa}$, Millipore, Spain, 3000 rpm centrifugation speed).

Elemental analysis of the $\mathrm{QD}_{\text {enr }}$ was performed using an ICP-MS equipped with a triple quadrupole (Agilent 8800, Agilent Technologies, Japan) by flow injection analysis (FIA). A two-position and six-ports injection valve (Rheodyne, IDEX Health \& Science, Germany) was used to allow on-line flow injection the $\mathrm{QD}_{\text {enr }}$ using the peristaltic pump of the instrument and ultrapure water as carrier. Operation conditions were daily optimized using a tuning solution and typical values are given in Table $\mathrm{S} 1$ (Appendix). $\mathrm{S}$ and $\mathrm{Cd}$ isotopes were detected using "mass shift mode" $\left({ }^{48} \mathrm{SO}^{+},{ }^{49} \mathrm{SO}^{+}\right.$and $\left.{ }^{50} \mathrm{SO}^{+}\right)$after their reaction with oxygen in the cell and "on mass mode" $\left({ }^{106} \mathrm{Cd}^{+}\right.$and $\left.{ }^{111} \mathrm{Cd}^{+}\right)$, respectively. Dwell time for each of the targeted isotopes was $100 \mathrm{~ms}$. Integration of FIA peaks was performed using the software provided with the instrument (MassHunter, Agilent).

\subsubsection{Determination of sulfur isotope ratios after correction for the mass bias.}

For determination of sulfur isotope ratios, mass bias was evaluated by measuring the corresponding ${ }^{34} \mathrm{~S} /{ }^{32} \mathrm{~S}$ ratio in a prepared $600 \mu \mathrm{g} \mathrm{L} \mathrm{L}^{-1}$ natural S ICP standard solution from a certified 1000 $\mathrm{mg} \mathrm{L}^{-1}$ standard (Merck, Germany).

A power law algorithm (Equation 1) was used for the correction of the mass bias (K) [19], typically observed in ICP-MS, due to the more efficient transmission of the heavier ions:

$$
\operatorname{Ln}\left(\frac{R_{\text {std }}}{R_{\text {theo }}}\right)=K \cdot \Delta M
$$

where $R_{\text {theo }}$ and $R_{\text {std }}$ are the theoretical (2009 IUPAC Technical Report [20]) and experimentally determined, in the natural sulfur abundance standard, ${ }^{34} \mathrm{~S} /{ }^{32} \mathrm{~S}$ isotope ratio, respectively. $\Delta \mathrm{M}$ is the mass difference between sulfur isotopes. Mass bias was determined daily obtaining a typical negative mass bias factor of $2-2.5 \%$ per mass unit.

Once calculated mass bias, it could be used for correction of the experimental ${ }^{34} \mathrm{~S} /{ }^{32} \mathrm{~S}$ isotope ratios obtained in target samples using the following equation:

$$
R_{c / k}=\frac{R_{S}}{e^{K \cdot \Delta M}}
$$


where $R_{s}$ and $R_{c / k}$ are the sulfur isotope ratios measured and corrected, respectively.

Finally, uncertainty propagation associated to the corrected isotope ratio (Equation 3), was calculated taking into account the uncertainty associated to the mass bias (Equation 4) as previously described elsewhere [21].

$$
\begin{aligned}
& {\left[\frac{s\left(R_{c / k}\right)}{R_{c / k}}\right]^{2}=\left[\frac{s\left(R_{S}\right)}{R_{S}}\right]^{2}+(-K \cdot \Delta M)^{2} \cdot\left[\frac{s(K)}{K}\right]^{2}} \\
& {\left[\frac{s(K)}{K}\right]^{2}=\left[\frac{1}{K \cdot \Delta M}\right]^{2} \cdot\left\{\left[\frac{s\left(R_{s t d}\right)}{R_{s t d}}\right]^{2}+\left[\frac{s\left(R_{\text {theo }}\right)}{R_{\text {theo }}}\right]^{2}\right\}}
\end{aligned}
$$

where $s\left(R_{c / k}\right)$, $s\left(R_{s t d}\right)$, $s\left(R_{s}\right)$ are the uncertainties for the corrected isotope ratio and the experimentally obtained isotope ratios in the natural abundance used standard for mass bias correction and in the sample, respectively. $s\left(R_{\text {theo }}\right)$ is the uncertainty associated to the natural sulfur isotope abundances [20]. Finally, $\mathrm{s}(\mathrm{K})$ is the uncertainty associated to the computation of the mass bias factor.

\subsubsection{Determination of sulfur isotopic abundances of the $Q D_{\text {enr. }}$.}

Isotopic abundance of the reference isotope in the $\mathrm{QD}_{\mathrm{enr}}$, (the most abundant ${ }^{34} \mathrm{~S}, A_{Q D}^{34}$ ) was first calculated using the following expression [22]:

$$
A_{Q D}^{34}=\frac{1}{\sum_{i=32}^{34} R\left(\frac{i}{34}\right)}
$$

where $R^{\frac{i}{34}}$ are the corrected isotope ratios for the different sulfur isotopes, $i$, regarding to the reference isotope measured in the sample (i.e. ${ }^{32} \mathrm{~S} /{ }^{34} \mathrm{~S},{ }^{33} \mathrm{~S} /{ }^{34} \mathrm{~S}$ and ${ }^{34} \mathrm{~S} /{ }^{34} \mathrm{~S}$ ), calculated as explained in Section 2.2.1. Their corresponding uncertainties $\mathrm{s}\left(R\left(\frac{i}{34}\right)\right)$ are calculated as explained in Section 2.2.1. The propagated uncertainty associated to $A_{Q D}^{34}, s\left(A_{Q D}^{34}\right)$, was calculated as follows [22]:

$$
\left[\frac{s\left(A_{Q D}^{34}\right)}{A_{Q D}^{34}}\right]^{2}=\sum_{i=32}^{34}\left[\left[\frac{R\left(\frac{i}{34}\right)}{\sum_{i=32}^{34} R\left(\frac{i}{34}\right)}\right]^{2} \cdot\left[\frac{s\left(R\left(\frac{i}{34}\right)\right)}{R\left(\frac{i}{34}\right)}\right]^{2}\right]
$$

Please note that $\mathrm{R}=1$ and $\mathrm{s}(\mathrm{R})=0$ for $\mathrm{i}=34$ by definition.

Isotopic abundances of minor isotopes of the element, $j$ (i.e. ${ }^{32} \mathrm{~S}$ and ${ }^{33} \mathrm{~S}$ ) were determined using: 


$$
A_{Q D}^{j}=A_{Q D}^{34} \cdot R\left(\frac{j}{34}\right)
$$

where $R^{\frac{j}{34}}$ is the corrected experimental isotope ratio of the minor isotopes regarding to the reference isotope (i.e. ${ }^{32} \mathrm{~S} /{ }^{34} \mathrm{~S},{ }^{33} \mathrm{~S} /{ }^{34} \mathrm{~S}$ ); $A_{Q D}^{j}$ is the isotopic abundance of such isotopes.

Regarding the uncertainty associated to the abundance of such minor isotopes, $s\left(A_{Q D}^{j}\right)$, it was calculated applying the following equation:

$$
\left[\frac{s\left(A_{Q D}^{j}\right)}{A_{Q D}^{j}}\right]^{2}=\left[\frac{s\left(A_{Q D}^{34}\right)}{A_{Q D}^{34}}\right]^{2}+\left[1-\frac{2 \cdot R\left(\frac{j}{34}\right)}{\sum_{i=32}^{34} R\left(\frac{i}{34}\right)}\right] \cdot\left[\frac{s\left(R\left(\frac{j}{34}\right)\right)}{R\left(\frac{j}{34}\right)}\right]^{2}
$$

where $s\left(R\left(\frac{j}{34}\right)\right)$ are the propagated uncertainties of the corresponding isotope ratios taking into account the mass bias correction as explained in the previous section. Thus, it is worth mentioning that $s\left(A_{Q D}^{34}\right)$ and $s\left(A_{Q D}^{j}\right)$ are the uncertainties associated to the isotopic abundances taking into account the whole isotope ratio measurement process.

\subsubsection{Determination of the number of $S$ moles per mol of water-soluble $Q D_{\text {enr. }}$}

Determination of the number of $\mathrm{S}$ moles per mol of $\mathrm{QD}_{\text {enr }}$ was performed following an indirect procedure based on previous $\mathrm{Cd}$ quantification [23]. First, $\mathrm{QD}_{\mathrm{enr}}$ were digested using aqua regia (15 minutes under ultrasonic bath) and Cd was quantified using external calibration prepared using certified $1000 \mathrm{mg} \mathrm{L}^{-1} \mathrm{Cd}$ standard (Merck, Germany). After Cd determination, computation of the elemental molar ratio $\mathrm{Cd} /{ }^{34} \mathrm{~S}$ in the $\mathrm{QD}_{\text {enr }}$ was determined by external FIA calibration using certified $1000 \mathrm{mg} \mathrm{L}^{-1} \mathrm{~S}$ and $\mathrm{Cd}$ standards (Merck). Cd concentration was kept constant while sulfur concentration was increased along the calibration curve as it can be seen in Fig. $3 \mathrm{a} \cdot{ }^{106} \mathrm{Cd} /{ }^{34} \mathrm{~S}$ peak area ratios observed in the $\mathrm{QD}_{\text {enr }}$ could be then translated into ${ }^{106} \mathrm{Cd} /{ }^{34} \mathrm{~S}$ molar ratios using the corresponding elemental calibration curve showed in the Fig. 3b. Such ${ }^{106} \mathrm{Cd} /{ }^{34} \mathrm{~S}$ molar ratio obtained could be easily translated into $\mathrm{Cd} / \mathrm{S}$ molar ratio using the $\mathrm{S}$ isotopic abundances computed as described in previous section 2.2.2 and the natural Cd isotopic abundances (2009 IUPAC Technical Report [20]). Finally, the number of S moles per mol of $\mathrm{QD}_{\text {enr }}$ could 
be computed taking into account the number of $\mathrm{Cd}$ moles present per mol of $\mathrm{QD}_{\text {enr }}$ and the $\mathrm{Cd} / \mathrm{S}$ molar ratio obtained.
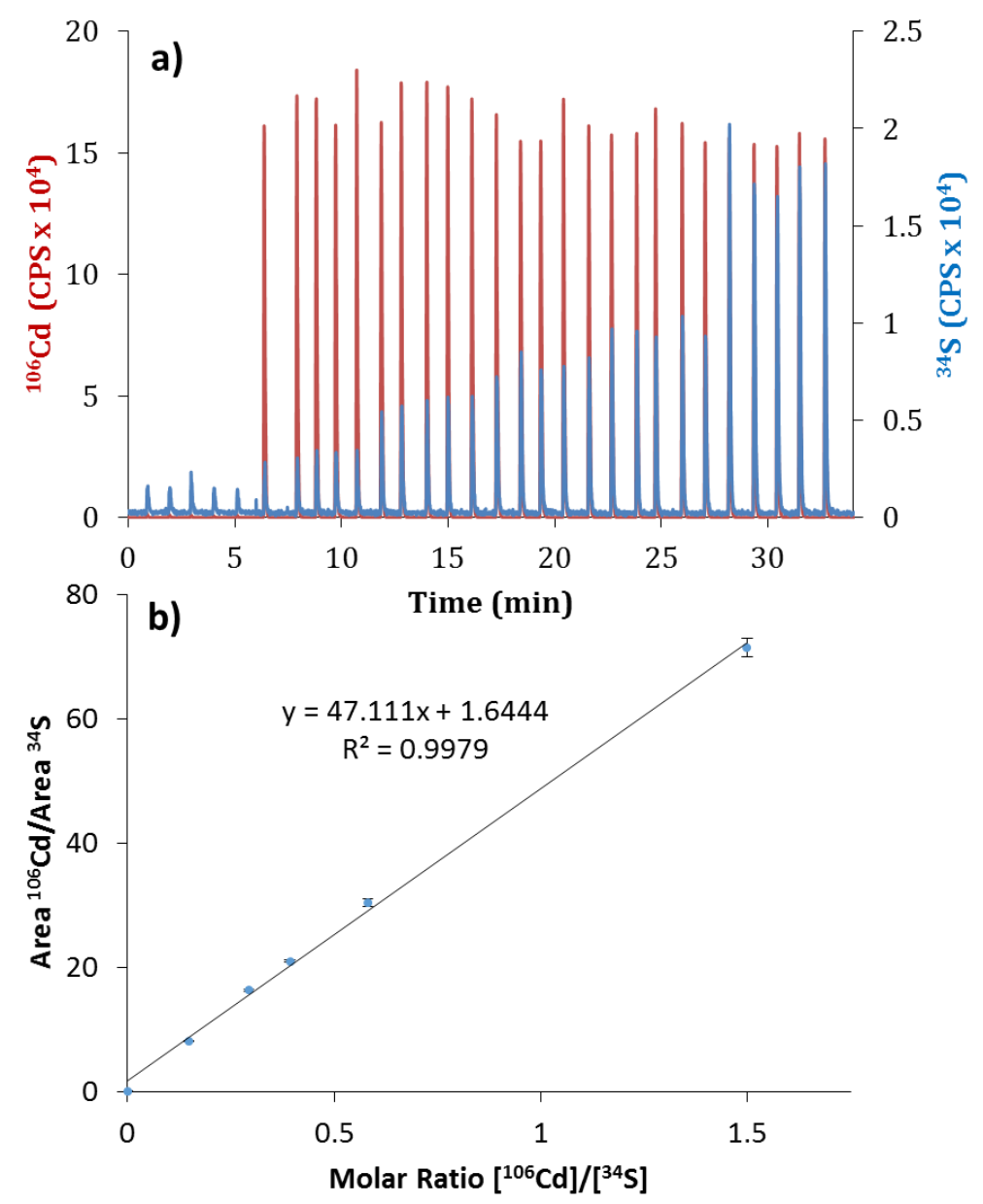

Figure 3. a) ICP-MS FIAgram obtained for the calculation of Cd:S molar ratios in the QDs: red and blue lines correspond to ${ }^{106} \mathrm{Cd}$ and ${ }^{34} \mathrm{~S} \mathrm{cps}$, respectively. Initial low intensity peaks observed $(0-5 \mathrm{~min})$ correspond to blank injections. b) Calibration plot for ${ }^{106} \mathrm{Cd}:{ }^{34} \mathrm{~S}$ area ratio versus ${ }^{106} \mathrm{Cd}:{ }^{34} \mathrm{~S}$ molar ratio. Error bars correspond to 1 standard deviation $(\mathrm{n}=5)$

\section{Results and discussion}

As typically carried out in typical species specific isotope dilution procedures, accurate elemental and isotopic characterization of the synthesized enriched species, in this case $\mathrm{QD}_{\mathrm{enr}}$, was first carried out [24]. Flow Injection Analysis (FIA) was selected for sample introduction since it provides higher sample throughput, lower sample consumption and minimize memory effects between samples. Additionally it 
allows simpler and more accurate correction of the S background. The ICP-MS/MS mass analyzer used allowed interference-free detection of the otherwise highly interfered target element $\mathrm{S}$, opening the door to accurate and precise measurement of the $\mathrm{S}$ isotope ratios, as peak area ratios [24-26]. Mass bias was corrected by measuring the ${ }^{34} \mathrm{~S} /{ }^{32} \mathrm{~S}$ ratio in a natural abundance $\mathrm{S}$ standard, injected $(\mathrm{n}=10)$ before the sample. A negative mass bias factor of 2-2.5\% per mass unit was obtained which was later used to correct the $\mathrm{S}$ isotope ratios computed in the $\mathrm{QD}_{\mathrm{enr}}$ solution $(\mathrm{n}=5)$. Isotopic composition of the synthesized $\mathrm{QD}_{\mathrm{enr}}$ is given in Table 1. As expected, high enrichment in ${ }^{34} \mathrm{~S}$ with the corresponding depletion in ${ }^{32} \mathrm{~S}$ were observed, (95.2 $\pm 0.1 \%$ and $3.7 \pm 0.1 \%$, respectively). Interestingly, isotope abundances of $\mathrm{QD}_{\text {enr }}$ are almost opposite to those observed in natural S. Of course, the small discrepancies observed between the isotopic ${ }^{32} \mathrm{~S}$ and ${ }^{34} \mathrm{~S}$ abundances of the enriched ${ }^{34} \mathrm{~S}_{8}$ used as starting material and those determined in the target $\mathrm{QD}_{\text {enr }}$ finally obtained are likely due to the presence of traces of natural $\mathrm{S}$ in the reagents and solvents used along the synthesis process.

Table 1. Isotope composition (\%) of the isotopically enriched $Q D_{\text {enr }}$ synthesized ( $n=10$; propagated uncertainty).

\begin{tabular}{|c|c|c|c|}
\hline Isotopes & $\begin{array}{c}\text { Natural S } \\
\text { abundances* }\end{array}$ & $\begin{array}{c}\text { Determined S abundances } \\
\text { in the } \text { QD }_{\text {enr }}\end{array}$ & $\begin{array}{c}\text { Reported S abundances } \\
\text { of the enriched }{ }^{34} \mathrm{~S}_{8}\end{array}$ \\
\hline${ }^{32} \mathrm{~S}$ & $94.99 \pm 0.26$ & $3.7 \pm 0.1$ & $>0.1$ \\
\hline${ }^{33} \mathrm{~S}$ & $0.75 \pm 0.02$ & $1.11 \pm 0.03$ & 98.8 \\
\hline${ }^{34} \mathrm{~S}$ & $4.25 \pm 0.24$ & $95.2 \pm 0.1$ & 1.1 \\
\hline
\end{tabular}

*Natural S abundances are taken from 2009 IUPAC Technical report [20].

Next, determination of the number of $\mathrm{S}$ atoms present per $\mathrm{QD}_{\mathrm{enr}}$ nanoparticle was performed by ICPMS. Acid digestion in aqua regia of the $\mathrm{QD}_{\mathrm{enr}}$ was first carried out. In order to avoid bias in the computation due to likely S contamination coming from the reagents used during the NP digestion as well as eventual S losses, an indirect approach was accomplished. First, number of $\mathrm{Cd}$ atoms per $\mathrm{QD}_{\text {enr }}$ were determined (210 $\pm 1 \mathrm{Cd}$ atoms/QD $\mathrm{enr}, \mathrm{n}=3$; uncertainty corresponds to $1 \mathrm{SD})$. This value was obtained from 
ICP-MS elemental analysis using external Cd calibration (see Experimental part and methods section) of a $\mathrm{QD}_{\text {enr }}$ solution previously digested, whose NP concentration had been previously calculated from the UV-Vis spectrum according to the Peng's equation [27]. Second, external FIA calibration was prepared by mixing increasing concentrations of a natural $\mathrm{S}$ standard with a fixed amount of a Cd natural standard and plotting the peak area ${ }^{106} \mathrm{Cd} /{ }^{34} \mathrm{~S}$ ratios experimentally obtained against the corresponding actual ${ }^{106} \mathrm{Cd} /{ }^{34} \mathrm{~S}$ molar ratios used (see Figure 3). In that way, the $\mathrm{Cd}$ to $\mathrm{S}$ molar ratios could be then easily computed in the $\mathrm{QD}_{\text {enr }}$ solution. Finally, taking into account such $\mathrm{Cd} / \mathrm{S}$ molar ratio value and the number of $\mathrm{Cd}$ atoms per $\mathrm{QD}_{\mathrm{enr}}$, a final value of $33 \pm 1 \mathrm{~S}$-enriched atoms per $\mathrm{QD}_{\mathrm{enr}}(\mathrm{n}=3$; uncertainty corresponds to $1 \mathrm{SD})$ could be obtained.

\subsection{Method development and calculations for quantitative assessment of nanoparticle:biomolecule} stoichiometry

Once $\mathrm{QD}_{\text {enr }}$ were synthesized and fully characterized, they could be applied for assessment of the number of sulfur-containing molecules (i.e. BSA, biotin) attached to a single $\mathrm{QD}_{\text {enr }}$ under different conditions. Mathematical treatment to extract sought biomolecule:NP stoichiometry from experimentally obtained sulfur isotope ratio measurements is similar to that of standard IDA approaches [28]. In this vein, after incubation of the $\mathrm{QD}_{\mathrm{enr}}$ with the investigated sulfur-containing analyte biomolecule (in the following referred to as "an"), the total sulfur moles of the mixture $\left(\mathbf{N S}_{\boldsymbol{m}}\right)$ can be expressed as the sum of sulfur moles from the analyte $\left(\mathbf{N S}_{\boldsymbol{a n}}\right)$ and the sulfur moles from the $\mathrm{QD}_{\mathrm{enr}}\left(\mathbf{N} \mathbf{S}_{\mathbf{Q D}}\right)$ :

$$
\mathbf{N} S_{m}=\mathbf{N S}_{a n}+\mathbf{N} S_{Q D}
$$

Such mass balance can be individually applied to isotopes ${ }^{34} \mathrm{~S}$ and ${ }^{32} \mathrm{~S}$, considering the actual isotope abundances of analyte and QD (see Table 1):

$$
\begin{aligned}
& \mathrm{N}^{34} S_{m}=\mathrm{N}^{34} S_{a n}+\mathrm{N}^{34} S_{Q D}=N S_{a n} \cdot A_{a n}^{34}+N S_{Q D} \cdot A_{Q D}^{34} \\
& \mathrm{~N}^{32} S_{m}=\mathrm{N}^{32} S_{a n}+\mathrm{N}^{32} S_{Q D}=N S_{a n} \cdot A_{a n}^{32}+N S_{Q D} \cdot A_{Q D}^{32}
\end{aligned}
$$


Dividing Eq. (10) by Eq. (11) Isotope Ratio $\left({ }^{34} \mathrm{~S} /{ }^{32} \mathrm{~S}\right)$ in the mixture $\left(\mathbf{R}_{\boldsymbol{m}}\right)$ is obtained, which can be directly and precisely measured by ICP-MS/MS:

$\mathbf{R}_{m}=\frac{\left(N S_{a n} \cdot A_{a n}^{34}+N S_{Q D} \cdot A_{Q D}^{34}\right)}{\left(N S_{a n} \cdot A_{a n}^{32}+N S_{Q D} \cdot A_{Q D}^{32}\right)}$

The number of mols of S coming from analyte $\left(\mathbf{N S}_{\boldsymbol{a n}}\right)$ and $\mathrm{QD}_{\mathrm{enr}}\left(\boldsymbol{N} \boldsymbol{S}_{\boldsymbol{Q D}}\right)$ can be expressed by taking into account the total number of mols of analyte $\left(\boldsymbol{N}_{\boldsymbol{a n}}\right)$ and $\mathrm{QD}_{\mathrm{enr}}\left(\boldsymbol{N}_{\boldsymbol{Q D}}\right)$ multiplied by the number of $\mathrm{S}$ mols present for each mol of analyte (S/an, known beforehand) and QD $_{\text {enr }}(\boldsymbol{S} / \mathbf{Q D}$, previously computed), respectively.

$\mathbf{R}_{m}=\frac{N_{a n} \cdot(S / a n) \cdot A_{a n}^{34}+N_{Q D} \cdot(S / Q D) \cdot A_{Q D}^{34}}{N_{a n} \cdot(S / a n) \cdot A_{a n}^{32}+N_{Q D} \cdot(S / Q D) \cdot A_{Q D}^{32}}$

After rearranging Eq. (13), number of mols of analyte attached to QD in the bioconjugate, that is to say stoichiometry of the nanoassembly, is given by:

$\frac{\mathbf{N}_{a n}}{N_{Q D}}=\frac{(S / Q D)}{(S / a n)} \cdot \frac{\left(A_{Q D}^{34}-\mathbf{R}_{m} \cdot A_{Q D}^{32}\right)}{\left(\mathbf{R}_{m} \cdot A_{a n}^{32}-A_{a n}^{34}\right)}$

From Eq. (14) it is clear the great potential of the proposed approach as the sought stoichiometry between the analyte and QD in the bioconjugate, can be directly obtained from a simple isotope ratio $\left({ }^{34} \mathrm{~S} /{ }^{32} \mathrm{~S}\right)$ measurement $\left(\mathbf{R}_{\boldsymbol{m}}\right)$ in the mixture. The rest of the parameters are known or have been determined previously during the characterization. Of course it is evident that the approach developed, as it is the case in any isotope dilution experiment, is limited by the value of $\mathbf{R}_{\boldsymbol{m}}$ experimentally measured. Indeed, it should be different enough from the starting $\left({ }^{34} \mathrm{~S} /{ }^{32} \mathrm{~S}\right)$ ratios of the individual components of the mixture, that is to say, the ratio of enriched $\mathrm{S}$ present in the $\mathrm{QD}_{\mathrm{enr}}(25.73 \pm 0.70)$ and the in the natural $\mathrm{S}$ present analyte $(0.0447 \pm 0.0015)$. Therefore, the most frequent relevant case where the number of $\mathrm{S}$ containing molecules surrounding the QD is high (i.e. protein corona), the minimum ${ }^{34} \mathrm{~S} /{ }^{32} \mathrm{~S}$ value 
measured in the QD-protein assembly that could be distinguishable at the $95 \%$ confidence level from the natural $\mathrm{S}$ isotope ratio is 0.0477 .

\subsection{Application to quantitative assessment of protein corona formation}

First application of the synthesized and characterized nanotool was to assess the average number of proteins that could surround it in a biological medium under different experimental conditions. It is well known from the bibliography that the number of proteins that could attach to the NP forming the so-called 'protein corona' can be high and well over one hundred [9]. BSA was selected as model protein in this case, as it is the most abundant protein in serum. Different mixtures containing increasing molar ratios of BSA to $\mathrm{QD}_{\text {enr }}$ were prepared ad incubated for $2 \mathrm{~h}$ (see Appendix). Since molecular weight of BSA is 66.4 $\mathrm{KDa}$, incubations were passed through a $100 \mathrm{KDa}$ cut-off centrifuge filter to eliminate the eventual excess of free BSA molecules that were not adsorbed-attached to the $\mathrm{QD}_{\mathrm{enr}}$ (see Appendix). It should be mentioned that it was previously demonstrated that efficiency of such filters to remove free BSA molecules in blank experiments was complete $(97 \pm 5 \%, \mathrm{n}=3)$. Finally, $\mathrm{S}$ isotope ratios in such purified incubated samples were measured by FIA with ICP-MS/MS detection. A typical FIAgram is given in Figure 4. Notably, less than 20 min were required to correct for mass bias and make replicate analyses of the sample. From Fig. 4a it is clear that peak area $\left({ }^{34} \mathrm{~S} /{ }^{32} \mathrm{~S}\right)$ ratio observed in the natural standard used to correct for mass bias was significantly different than that observed in the $\mathrm{QD}_{\text {enr }}$ surrounded by BSA (Fig.4b). 


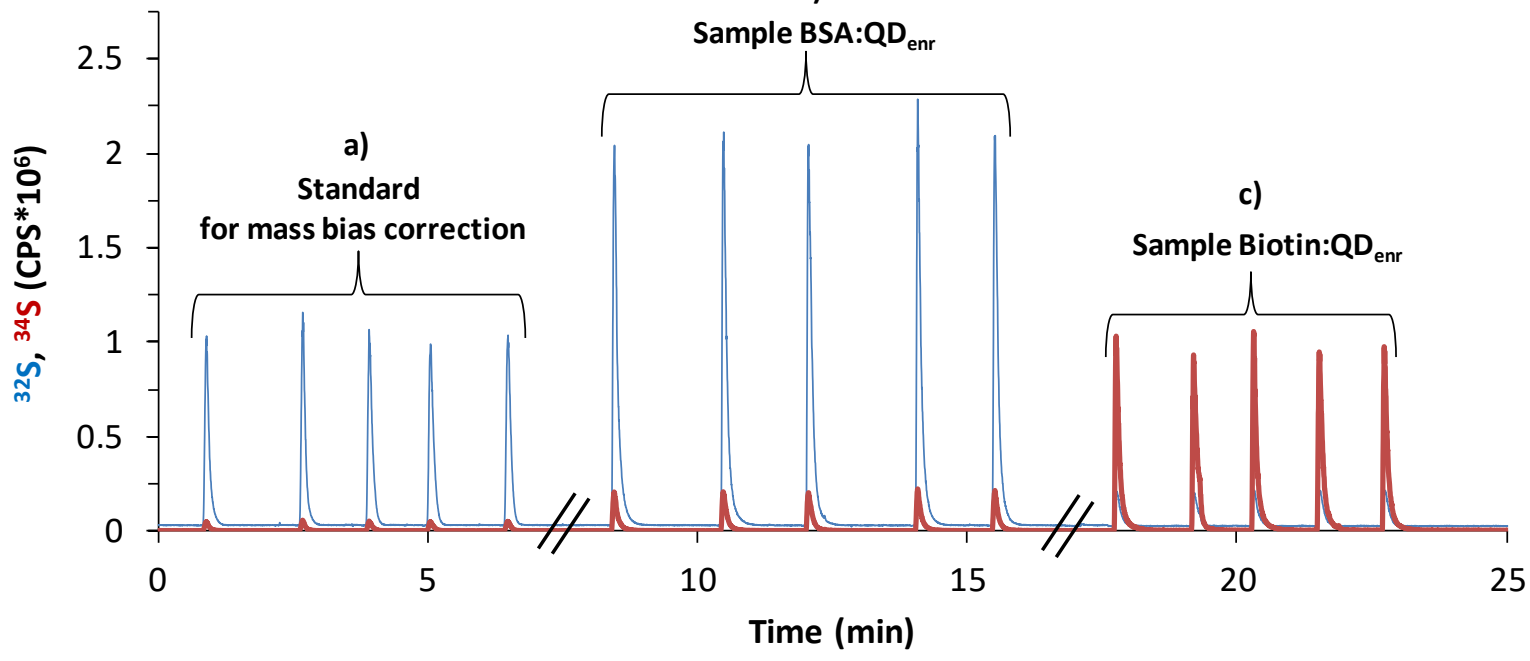

Figure 4. Examples of ICP-MS FIAgrams obtained for the analysis of: a) S ICP standard use for mass bias correction, b) sample obtained after incubation of $Q D_{\text {enr }}$ with $B S A$ with a $B S A: Q D_{\text {enr }}$ molar ratio 27:1 and c) sample obtained after bioconjugation of $Q D_{\text {enr }}$ with biotin with a Biotin:QDenr molar ratio 12:1.

Once $\mathbf{R}_{\boldsymbol{m}}$ was experimentally obtained, direct application of equation 14, enabled us to calculate the sought BSA:QDenr (protein corona) stoichiometry. Results are shown in Table 2 where the individual corrected $\mathbf{R}_{\boldsymbol{m}}$ values with their corresponding propagated uncertainty (around 3\% RSD, always limited by the uncertainty on the values of sulphur natural isotopic abundances, used for the mass bias correction [19]) are also given.

Table 2. Stoichiometry obtained for the different BSA:QDenr incubations (protein corona) assayed ( $n=5$; propagated uncertainty).

\begin{tabular}{|c|c|c|}
\hline \multicolumn{2}{|c|}{ Molar Ratio (BSA:QD } & \\
\hline $\begin{array}{c}\text { Assayed } \\
\text { (theoretical) }\end{array}$ & $\begin{array}{c}\text { Obtained } \\
\text { (experimental) }\end{array}$ & $\mathbf{R}_{\mathbf{m}}$ \\
\hline 27 & $15 \pm 1$ & $0.100 \pm 0.003$ \\
\hline 83 & $28 \pm 3$ & $0.0748 \pm 0.0025$ \\
\hline 83 & $32 \pm 3$ & $0.0713 \pm 0.0023$ \\
\hline
\end{tabular}




\begin{tabular}{|c|c|c|}
\hline 83 & $28 \pm 3$ & $0.0752 \pm 0.0025$ \\
\hline 353 & $111 \pm 33$ & $0.0524 \pm 0.0017$ \\
\hline 1404 & $221 \pm 151$ & $0.0486 \pm 0.0016$ \\
\hline
\end{tabular}

Uncertainty propagation associated with the final BSA: $\mathrm{QD}_{\mathrm{enr}}$ stoichiometry was carried out using the Kragten spreadsheet method [29]. Values obtained were excellent ranging from 6 to $10 \%$ RSD for the protein:NP ratios below 100. It is worth noting that this is a quite challenging situation because the number of natural ${ }^{32} \mathrm{~S}$ atoms incorporated to the nanostructure $\mathrm{BSA}: \mathrm{QD}_{\mathrm{enr}}$ through the individual protein molecules is very high in comparison with the number of enriched ${ }^{34} \mathrm{~S}$ atoms present in the $\mathrm{QD}_{\text {enr, }}$ even at comparatively low BSA:QDenr ratios. This is why measured $\mathrm{R}_{\mathrm{m}}$ values approach progressively natural $\mathrm{S}$ value as the number of BSA molecules in the protein corona increases: the denominator of equation (14) becomes very small and $R_{m}$ values $(0.0524 \pm 0.0017$ and $0.0486 \pm 0.0016$, respectively) get really close to the natural $\left({ }^{34} \mathrm{~S} /{ }^{32} \mathrm{~S}\right)$ ratio. An immediate result is a worsening precision to 30 and $68 \%$ RSD when the number of proteins of the corona is 111 and 221, respectively. This is consistent with the limits established in section 3.1. Of course, number of proteins that can be precisely (RSD $<10 \%$ ) determined in the corona using this approach could be significantly extended just by increasing the size of the shell during the QD synthesis (i.e. the number of S atoms present in each QD).

In addition, the repeatability of the approach was tested by measuring three different incubations with the same starting protein molar excess (83). As can be seen in Table 2, the agreement between individual incubation replicates was also excellent with an inter-assay precision around $8 \% \mathrm{RSD}$, in the level of the intra-assay precision of the individual experiments.

\subsection{Application to quantitative assessment of the conjugation between nanoparticles and biomolecules}

Second application of the synthesized and characterized nanotool was the accurate determinations of the number of sulfur containing molecules chemically bound to the surface of a given engineered $\mathrm{QD}_{\mathrm{enr}}$. The impact of such potential application is enormous as there are a large number of S-containing small molecules currently used to solubilize and functionalize QDs including cysteine, DHLA (dihydrolipoic 
acid) and MPA (mercaptopropionic acid), among many others. Of course, accurate control of the ligand density in such reactions is a key parameter affecting further nanoparticle labeling and bioavailability features.

In this case we selected biotin, extensively used to establish a highly specific affinity noncovalent interaction with streptavidin. Such strong biotin-streptavidin interaction enables the development of selective NP-based assays, particularly immunoassays [30]. Carbodiimide chemistry (EDC) [30] was used to establish a chemical bond between the carboxyl groups of the amphiphilic polymer of the soluble $\mathrm{QD}_{\mathrm{enr}}$ surface and the amine group of the biotin hydrazide molecule, which also contains $1 \mathrm{~S}$ atom. After bioconjugation, samples were purified again by ultracentrifugation to eliminate eventual free biotin molecules excess (see Appendix for the details). Again, blank experiments were first performed to demonstrate that efficiency of such ultrafiltration approach to remove free biotin molecules was complete $(98 \pm 2 \%, n=3)$. Sulfur isotope ratios were then computed as peak area ratios in the corresponding FIAgram and corrected for mass bias as explained before for BSA experiments. Typical FIAgram peaks are shown in Fig. 4c. As can be clearly seen by the high enrichment in ${ }^{34} \mathrm{~S}, \mathrm{R}_{\mathrm{m}}$ observed was this time far from the natural $\mathrm{S}$ isotope ratio and closer to the enriched $\mathrm{S}$ one present in the $\mathrm{QD}_{\mathrm{enr}}$. Once more, simple application of equation (14) provided directly the experimental stoichiometry biotin: $\mathrm{QD}_{\mathrm{enr}}$ for each individual conjugation. Results are given in Table 3.

Table 3. Stoichiometry obtained for the different Biotin: $Q D_{\text {enr }}$ incubations assayed ( $n=5$; propagated uncertainty).

\begin{tabular}{|c|c|c|}
\hline \multicolumn{2}{|c|}{ Molar Ratio (Biotin:QD } & \multirow{2}{*}{$\mathbf{R}_{\mathbf{m}}$} \\
\hline $\begin{array}{c}\text { Assayed } \\
\text { (theoretical) }\end{array}$ & $\begin{array}{c}\text { Obtained } \\
\text { (experimental) }\end{array}$ & \\
\hline 4.3 & $4.5 \pm 0.3$ & $5.7 \pm 0.2$ \\
\hline 5.3 & $5.3 \pm 0.3$ & $5.0 \pm 0.2$ \\
\hline 6.3 & $6.2 \pm 0.3$ & $4.4 \pm 0.1$ \\
\hline 7.7 & $5.0 \pm 0.3$ & $5.2 \pm 0.2$ \\
\hline
\end{tabular}




\begin{tabular}{|c|c|c|}
\hline 9.2 & $6.1 \pm 0.3$ & $4.5 \pm 0.1$ \\
\hline 12 & $5.5 \pm 0.3$ & $4.9 \pm 0.2$ \\
\hline 19 & $5.4 \pm 0.3$ & $4.9 \pm 0.2$ \\
\hline 41 & $5.0 \pm 0.3$ & $5.3 \pm 0.2$ \\
\hline 81 & $5.2 \pm 0.3$ & $5.1 \pm 0.2$ \\
\hline
\end{tabular}

Propagated uncertainty associated with the obtained biotin: $\mathrm{QD}_{\mathrm{enr}}$ stoichiometry was again excellent (6$11 \% \mathrm{RSD}$ ). It is interesting to note that for the eight biotin:QDenr molar ratios assayed above 5 (ranging from 5.3 to 81), molar ratios experimentally obtained ranged randomly between 5 and 6 (average value is $5.5 \pm 0.5, \mathrm{n}=8)$. In fact, stoichiometry obtained turned out to be below $5(4.5 \pm 0.3)$ only when an initial molar ratio of 4.3 was assayed. It seems that steric effects hinder the formation of more than 6 amide chemical bonds between biotin molecules and the carboxyl groups present on the $\mathrm{QD}_{\mathrm{enr}}$ surface. The individual biotin molecules likely need to reach the QD surface under the correct orientation to form the chemical bond, which limits significantly the maximum stoichiometry achievable. These results are in excellent agreement with the value of biotin loading on the surface of commercial QD conjugates that typically ranges from 5 to 7 [31]. This controlled and stoichiometric process contrasts with the formation of the 'protein corona', where QD works as a seed for the growing of the protein-protein interactions steered by electrostatic forces (i.e. hydrophobic effect).

\section{Conclusions}

In brief, direct determination of stoichiometry between QDs and different S-containing biomolecules can be quantitatively assessed by resorting to ${ }^{34}$ S-enriched QDs and a single precise isotope ratio measurement. Neither methodological calibrations nor complex sample preparation procedures are required. It should be noted that, as compared to existing indirect methods based on quantification of unbound proteins, here-proposed approach provides the biomolecule:nanoparticle ratio in the final nanoassembly without the need to know in advance the concentration of the biomolecule in the starting 
solution, a parameter many often difficult to know. Notably, there is also no need for previous labeling of the biomolecules with fluorescent or radioactive labels. Indeed, isotopically enriched nanotool designed here can be used for studying interactions with many different S-containing biomolecule without specific labelling for each biomolecule under study.

Two main application fields are clearly foreseen here. First is the optimization of the procedure to produce functionalized NPs with applicability in NP-based immunoassays, imaging and trafficking studies or drug delivery systems. Highly precise sulfur isotope ratio measurement by ICP-MS would allow direct and fast control of the resulting bioconjugates, obtained under different experimental conditions. Since the $\mathrm{QD}_{\mathrm{enr}}$ is identical to the natural $\mathrm{QDs}$, once the protocol has been optimized and is fully under control, production of the desired conjugate using natural QDs could be easily performed.

Second application field consists of the in vivo and in vitro assessment of the protein corona formation. Different procedures for QD water solubilization and functionalization could be assayed in different biological media. Again, direct measurement of the isotope ratio will easily allow the precise quantification of the number of proteins constituting the protein corona (even studies of the soft and hard corona would be possible).

\section{Acknowledgments}

We would like to acknowledge the financial support provided by the Spanish Ministry of Education (CTQ2016-79412-P) from Asturias Regional Government (Projects FC-15-GRUPIN14-092 and FC-15GRUPIN14-106) and Agilent Technologies Foundation. M. Menendez Miranda acknowledges the Ph.D. grant (BP12-46) from Asturias Regional Government (Spain) and D. Presa Soto acknowledges the Ph.D. grant from Spanish Ministry of Education (Spain). A. Presa Soto would like to thank the MEC for the Ramón y Cajal program.

\section{Supplementary data to this article can be found online at doi:...}




\section{References}

[1] A.M. Coto-García, E. Sotelo-González, M.T. Fernández-Argüelles, R. Pereiro, J.M. CostaFernández, A. Sanz-Medel, Nanoparticles as fluorescent labels for optical imaging and sensing in genomics and proteomics, Anal. Bioanal. Chem. 399 (2011) 29-42. doi:10.1007/s00216-0104330-3.

[2] W.R. Algar, K. Susumu, J.B. Delehanty, I.L. Medintz, Semiconductor Quantum Dots in Bioanalysis: Crossing the Valley of Death, Anal. Chem. 83 (2011) 8826-8837. doi:10.1021/ac201331r.

[3] C.-A.J. Lin, T. Liedl, R.A. Sperling, M.T. Fernández-Argüelles, J.M. Costa-Fernández, R. Pereiro, A. Sanz-Medel, W.H. Chang, W.J. Parak, Bioanalytics and biolabeling with semiconductor nanoparticles (quantum dots), J. Mater. Chem. 17 (2007) 1343-1346. doi:10.1039/B618902D.

[4] J.B. Delehanty, K. Boeneman, C.E. Bradburne, K. Robertson, I.L. Medintz, Quantum dots: a powerful tool for understanding the intricacies of nanoparticle-mediated drug delivery, Expert Opin. Drug Deliv. 6 (2009) 1091-1112. doi:10.1517/17425240903167934.

[5] P. Del Pino, B. Pelaz, Q. Zhang, P. Maffre, G.U. Nienhaus, W.J. Parak, Protein corona formation around nanoparticles - from the past to the future, Mater. Horiz. 1 (2014) 301-313. doi:10.1039/C3MH00106G.

[6] C.D. Walkey, W.C.W. Chan, Understanding and controlling the interaction of nanomaterials with proteins in a physiological environment, Chem. Soc. Rev. 41 (2012) 2780-2799. doi:10.1039/C1CS15233E.

[7] C.D. Walkey, J.B. Olsen, F. Song, R. Liu, H. Guo, D.W.H. Olsen, Y. Cohen, A. Emili, W.C.W. Chan, Protein Corona Fingerprinting Predicts the Cellular Interaction of Gold and Silver Nanoparticles, ACS Nano. 8 (2014) 2439-2455. doi:10.1021/nn406018q.

[8] C. Röcker, M. Pötzl, F. Zhang, W.J. Parak, G.U. Nienhaus, A quantitative fluorescence study of protein monolayer formation on colloidal nanoparticles, Nat. Nanotechnol. 4 (2009) 577-580. doi:10.1038/nnano.2009.195. 
[9] N. Fernández-Iglesias, J. Bettmer, Complementary mass spectrometric techniques for the quantification of the protein corona: a case study on gold nanoparticles and human serum proteins, Nanoscale. 7 (2015) 14324-14331. doi:10.1039/C5NR02625C.

[10] X. Zhao, D. Lu, F. Hao, R. Liu, Exploring the diameter and surface dependent conformational changes in carbon nanotube-protein corona and the related cytotoxicity, J. Hazard. Mater. 292 (2015) 98-107. doi:10.1016/j.jhazmat.2015.03.023.

[11] D.-H. Tsai, F.W. DelRio, A.M. Keene, K.M. Tyner, R.I. MacCuspie, T.J. Cho, M.R. Zachariah, V.A. Hackley, Adsorption and Conformation of Serum Albumin Protein on Gold Nanoparticles Investigated Using Dimensional Measurements and in Situ Spectroscopic Methods, Langmuir. 27 (2011) 2464-2477. doi:10.1021/la104124d.

[12] Q. Peng, S. Zhang, Q. Yang, T. Zhang, X.-Q. Wei, L. Jiang, C.-L. Zhang, Q.-M. Chen, Z.-R. Zhang, Y.-F. Lin, Preformed albumin corona, a protective coating for nanoparticles based drug delivery system, Biomaterials. 34 (2013) 8521-8530. doi:10.1016/j.biomaterials.2013.07.102.

[13] B. Meermann, K. Wichmann, F. Lauer, F. Vanhaecke, T.A. Ternes, Application of stable isotopes and AF4/ICP-SFMS for simultaneous tracing and quantification of iron oxide nanoparticles in a sediment-slurry matrix, J. Anal. At. Spectrom. 31 (2016) 890-901. doi:10.1039/C5JA00383K.

[14] J. Gigault, V.A. Hackley, Differentiation and characterization of isotopically modified silver nanoparticles in aqueous media using asymmetric-flow field flow fractionation coupled to optical detection and mass spectrometry, Anal. Chim. Acta. 763 (2013) 57-66. doi:10.1016/j.aca.2012.11.060.

[15] A. Laycock, B. Stolpe, I. Römer, A. Dybowska, E. Valsami-Jones, J.R. Lead, M. Rehkämper, Synthesis and characterization of isotopically labeled silver nanoparticles for tracing studies, Environ. Sci. Nano. 1 (2014) 271. doi:10.1039/c3en00100h.

[16] M. Pálmai, R. Szalay, D. Bartczak, Z. Varga, L.N. Nagy, C. Gollwitzer, M. Krumrey, H. GoenagaInfante, Total synthesis of isotopically enriched $\mathrm{Si}-29$ silica NPs as potential spikes for isotope dilution quantification of natural silica NPs, J. Colloid Interface Sci. 445 (2015) 161-165. 
doi:10.1016/j.jcis.2014.12.085.

[17] Z.A. Peng, X. Peng, Formation of High-Quality CdTe, CdSe, and CdS Nanocrystals Using CdO as Precursor, J. Am. Chem. Soc. 123 (2001) 183-184. doi:10.1021/ja003633m.

[18] M.T. Fernández-Argüelles, A. Yakovlev, R.A. Sperling, C. Luccardini, S. Gaillard, A. Sanz Medel, J.-M. Mallet, J.-C. Brochon, A. Feltz, M. Oheim, W.J. Parak, Synthesis and Characterization of Polymer-Coated Quantum Dots with Integrated Acceptor Dyes as FRETBased Nanoprobes, Nano Lett. 7 (2007) 2613-2617. doi:10.1021/n1070971d.

[19] I.S. Begley, B.L. Sharp, Characterisation and Correction of Instrumental Bias in Inductively Coupled Plasma Quadrupole Mass Spectrometry for Accurate Measurement of Lead Isotope Ratios, J. Anal. At. Spectrom. 12 (1997) 395-402. doi:10.1039/a605078f.

[20] M. Berglund, M.E. Wieser, Isotopic compositions of the elements 2009 (IUPAC Technical Report), Pure Appl. Chem. 83 (2011) 397-410. doi:10.1351/PAC-REP-10-06-02.

[21] J.Á. Rodríguez-Castrillón, M. Moldovan, J. Ruiz Encinar, J.I. García Alonso, Isotope pattern deconvolution for internal mass bias correction in the characterisation of isotopically enriched spikes, J. Anal. At. Spectrom. 23 (2008) 318-324. doi:10.1039/B710595A.

[22] J.G. Alonso, Determination of fission products and actinides by inductively coupled plasma-mass spectrometry using isotope dilution analysis: A study of random and systematic errors, Anal. Chim. Acta. 312 (1995) 57-78. doi:10.1016/0003-2670(95)00199-A.

[23] M. Menendez-Miranda, M.T. Fernandez-Arguelles, J.M. Costa-Fernandez, J.R. Encinar, A. SanzMedel, Elemental ratios for characterization of quantum-dots populations in complex mixtures by asymmetrical flow field-flow fractionation on-line coupled to fluorescence and inductively coupled plasma mass spectrometry, Anal. Chim. Acta. 839 (2014) 8-13. doi:10.1016/j.aca.2014.06.034.

[24] S.D. Fernández, N. Sugishama, J.R. Encinar, A. Sanz-Medel, Triple Quad ICPMS (ICPQQQ) as a New Tool for Absolute Quantitative Proteomics and Phosphoproteomics, Anal. Chem. 84 (2012) 5851-5857. doi:10.1021/ac3009516. 
[25] L. Balcaen, G. Woods, M. Resano, F. Vanhaecke, Accurate determination of S in organic matrices using isotope dilution ICP-MS/MS, J. Anal. At. Spectrom. 28 (2013) 33-39. doi:10.1039/C2JA30265A.

[26] F. Calderón-Celis, S. Diez-Fernández, J.M. Costa-Fernández, J.R. Encinar, J.J. Calvete, A. SanzMedel, Elemental Mass Spectrometry for Absolute Intact Protein Quantification without ProteinSpecific Standards: Application to Snake Venomics, Anal. Chem. 88 (2016) 9699-9706. doi:10.1021/acs.analchem.6b02585.

[27] W.W. Yu, L. Qu, W. Guo, X. Peng, Experimental Determination of the Extinction Coefficient of CdTe, CdSe, and CdS Nanocrystals, Chem. Mater. 15 (2003) 2854-2860. doi:10.1021/cm034081k.

[28] J.I. Garcia-Alonso, P. Rodrigez-Gonzalez, Isotope dilution mass spectrometry, Royal Society of Chemistry, Cambridge, UK, 2013.

[29] J. Kragten, Tutorial review. Calculating standard deviations and confidence intervals with a universally applicable spreadsheet technique, Analyst. $119 \quad$ (1994) 2161. doi:10.1039/an9941902161.

[30] G. Hermanson, Bioconjugate Techniques, Elsevier, USA, 1996.

[31] Qdot $®$ Biotin Conjugates User Manual Qdot @ Biotin Conjugates User Manual, (n.d.). 